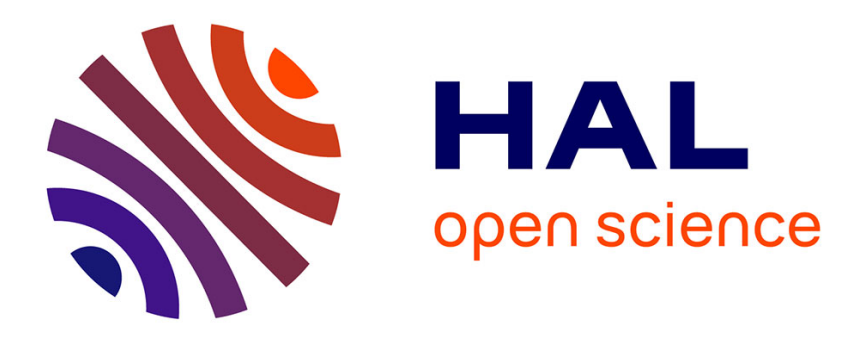

\title{
Enhanced Unequal Clustering Algorithm for Wireless Sensor Networks
}

Said Talbi, Lotfi Zaouche

\section{To cite this version:}

Said Talbi, Lotfi Zaouche. Enhanced Unequal Clustering Algorithm for Wireless Sensor Networks. 12th ACS/IEEE International Conference on Computer Systems and Applications (AICCSA 2015), Nov 2015, Marrakech, Morocco. 10.1109/AICCSA.2015.7507116 . hal-01378481

\section{HAL Id: hal-01378481 \\ https://hal.science/hal-01378481}

Submitted on 13 Oct 2016

HAL is a multi-disciplinary open access archive for the deposit and dissemination of scientific research documents, whether they are published or not. The documents may come from teaching and research institutions in France or abroad, or from public or private research centers.
L'archive ouverte pluridisciplinaire HAL, est destinée au dépôt et à la diffusion de documents scientifiques de niveau recherche, publiés ou non, émanant des établissements d'enseignement et de recherche français ou étrangers, des laboratoires publics ou privés. 


\section{Enhanced Unequal Clustering Algorithm for Wireless Sensor Networks}

\author{
Said Talbi \\ University of Tizi Ouzou, Algeria \\ Department of Computer Science \\ Email: s_talbi@esi.dz
}

\author{
Lotfi Zaouche \\ Sorbonne universités, Université de technologie de Compiègne, \\ CNRS, UMR 7253 Heudiasyc \\ - CS 60319 - 60203 Compiègne cedex \\ Email: lotfi.zaouche@hds.utc.fr
}

\begin{abstract}
Clustering is considered as solution for more energy conservation during communications in wireless sensor networks. Recently, a new clustering algorithm named Unequal Clustering Algorithm (UCA) is proposed to avoid the burdened clusterheads located around the sink due to the traffic coming from others which are far to the base station. This paper presents an Enhanced Unequal Clustering Algorithm called EUCA. This solution reduces the control traffic during a clustering process by eliminating the competition of nodes to become cluster-head and allows a better reactivity to event. Simulation result shows that EUCA conserves more energy.
\end{abstract}

\section{Problem Statement}

Rapid technological advances in the fields of MEMS (Micro Electro-Mechanical Systems) and low-power radio frequency design have led to the birth of Wireless Sensor Networks (WSNs). A WSN has been found to have big potential for wide applications such as disaster management, environment monitoring, health care, defense reconnaissance, etc [1]. A WSN is composed of hundreds of small sensor nodes that are deployed in a given coverage area. All the sensor nodes collect local data, process and send it to a remote base station.

The sink is connected to the Internet for the public notice of the phenomena. Sensor nodes carry limited or irreplaceable power sources, and in many scenarios, it is difficult to replace the sensor nodes after complete depletion of their energy. Therefore, reducing energy consumption of the sensor nodes for maximizing network lifetime is considered as the most critical challenge in WSN. Many research issues have been addressed on this topic which can be found in the survey paper [2]. Energy efficient clustering design is one of these issues. In a cluster based WSN, the sensor nodes are grouped into distinct clusters with a single leader for each cluster, called cluster-head (see Fig. 1). Cluster-heads are generally selected among normal sensor nodes. In some cases, nodes with high energy capacity called gateways are deployed as cluster-heads [3], [4]. Each sensor node belongs to one and only one cluster and communicates with its cluster-head. The sensor nodes sense local data and send it to their clusterhead. After receiving data, the cluster-heads perform data aggregation and send processed data to the sink through other cluster-heads. If cluster-heads are selected from normal sensor nodes, they can die quickly due to the extra work load for data aggregation and forwarding. Moreover, cluster-heads closer to the sink are inevitably used as a relays to forward data packets, and thereby drain their energy very quickly. Therefore, the clustering process and the data routing need to be properly achieved for balancing the energy consumption of the clusterheads to improve the network lifetime. To achieve this, Chen proposed Unequal Clustering Algorithm (UCA) [5] which considers that clusters around the sink should be smaller, because the headers of these clusters consume more energy due to the extra load when forwarding data of cluster-heads which are far to the base station.

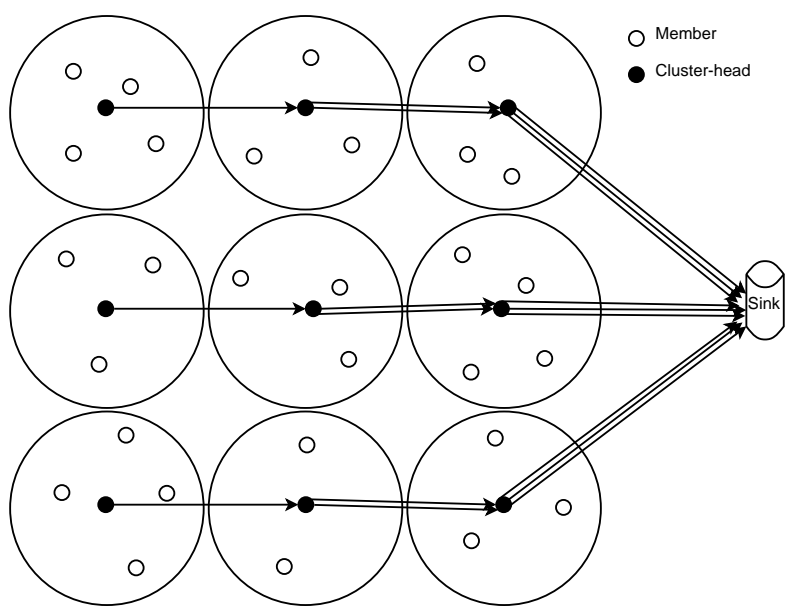

Fig. 1. Wireless Sensor Network Model

In this paper, we present an Enhanced Unequal Algorithm (EUCA). This algorithm reduces the number of exchanged control messages during cluster-heads election. The remainder of this paper is structured as follows. Section II summarizes related work on the clustering algorithms. Section III describes the considered network and the model of the energy consumption. Section IV gives a description of UCA. Section V presents EUCA. Section VI discusses the results of performance evaluation. Finally, Section VII conclude the paper with future direction work.

\section{RELATED WORK}

LEACH [6] (Low Energy Adaptive Hierarchy) is one of the most popular cluster based routing technique. It uses randomized rotation of cluster-heads to distribute energy consumption over all nodes in the network. However, this protocol presents 
some inconvenient such as: sensor node with low energy may be selected as a cluster-head, the distribution of cluster-heads are not uniform through the network. Many works have been presented in the literature in order to improve LEACH [7]-[9]

HEED [10] (Hybrid Energy Efficient Distributed Protocol) periodically selects cluster-hea ds based on the node's residual energy and proximity measure of the neighbor nodes or node degree, and introduces a variable transmission power to be used for intra-cluster communication. HEED uses the shortest path in terms of hop counts for inter-cluster communication, which is not necessarily the reliable and energy efficient.

Zhang et al. [11] proposed VLCA (maximum-Votes and Load-balance Clustering Algorithm). Each sensor node collects votes from their neighbors and calculates the total vote received. The more votes a sensor accumulates, the more important it is in the whole network. During the clustering phase, sensors compete with each other based on the total votes each has received. This approach leads to a considerable exchanging messages

In some works, the occurrence of an event leads to the formation of a cluster [12]-[14]. In this case, routing protocol needs to form only one cluster with one header for minimizing the amount of data forwarding to the base station. These approaches are event based clustering protocols and may generate a delay to report the phenomena.

Chen et al. [5] proposed UCA which considers that the size (i.e., number of sensor nodes members ) of clusters around the sink should be smaller, because the headers of these clusters consume more energy due to the data traffic coming from other cluster-heads located far to the sink. Unlike LEACH, this protocol allows a good distribution of cluster-heads through the network by adopting a competition to designate clusterheads rather than a self- election. However, the competition performed in UCA causes significant exchange of control messages.

\section{Network And EnERgy COnsumption Model}

\section{A. Network Model}

The network assumed is a square sensing area, and $\mathrm{N}$ sensor nodes randomly scattered within this area. The following hypothesis give an idea about the sensor nodes and the network taken into consideration :

1) The network contains a unique sink which is stationary

2) All the sensor nodes are stationary and homogeneous

3) The power control is used by all sensor nodes to adjust the transmission range

4) The sensors nodes are all reachable by the sink. Latter is responsible for launching the clustering process (i.e., new rounds)

\section{B. Energy Consumption Model}

To evaluate the energy spent when communicating, we use the model proposed by Heinzelman et al. [6]. The energy $E_{T X}(k, d)$ consumed to transmit a $k$-bit packet over distance $d$ is :

$$
E_{T X}(k, d)=k \times\left(E_{t r}+E_{a m p} d^{\alpha}\right)
$$

where $E_{t r}$ is the energy required by the electronic transceiver to transmit one bit; $E_{a m p}$ is the energy amplification per bit; $\alpha$ is a constant which depends on the signal attenuation, it is equal to 2 (i.e., free space model) or 4 (i.e., multi-path fading model) [6]. In addition, the radio consumption energy $E_{R X}$ in reception for the same packet is :

$$
E_{R X}(k)=k \times E_{t r}
$$

\section{Unequal Clustering Algorithm}

The main issue in WSNs is to increase the network lifetime under energy limitation of sensors. To achieve this goal, energy dissipation must be effectively balanced between all nodes. The role of cluster-head in homogeneous networks is generally turned among nodes to balance the energy consumption. Though, the avoiding of energy hole problem cannot completely achieved. Indeed, the rotation of the cluster-heads role balances the energy consumption among the headers and member nodes, thereby, it could hardly fulfill those above among cluster-heads during the inter-cluster communications. Since nodes around the sink deplete quickly their energy resource due to traffic coming from others nodes which are far to the sink.

In UCA [5], the size of clusters around the sink should be smaller, because the headers of these clusters consume more energy. So more clusters should be formed closer to the base station (see Fig. 2). To do this, the node's competition radius range should be proportional to is the distance between the node and the sink. Consider $R_{\max }$ the greatest competition radius that is predefined. The node $i$ calculates its competition radius range $R_{i}$ based on its distance to the sink as follow :

$$
R_{i}=\left(1-c \times \frac{d_{\max }-d(i, S B)}{d_{\max }-d_{\min }}\right) \times R_{\max }
$$

where $d(i, S B)$ is a distance between node $i$ and the base station, $d_{\min }$ is the distance between the nearest node and the base station, $d_{\max }$ is distances between the farthest node to the base station and $c$ is a constant belonging the interval $[0,1]$. By the equation (3), the competition radius range takes its values between $(1-c) R_{\max }$ and $R_{\max }$.

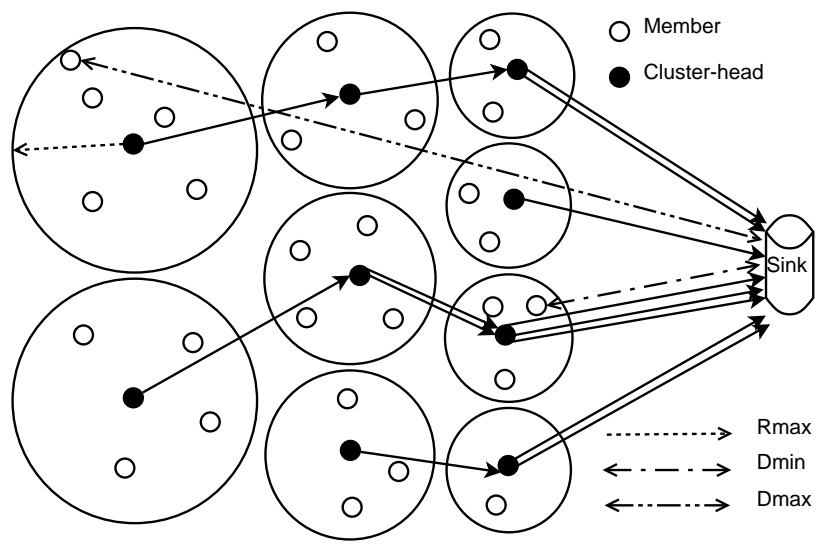

Fig. 2. An overview of the unequal clustering mechanism [5] 
During the phase of cluster-heads election, the nodes exchange their energy residual using appropriate competition radius. A node which have the greatest amount energy decides to become cluster-head for the current round. This mechanism avoid to elect more than one cluster-head on the same region of competition. However, the number of exchanging messages is not negligible. Indeed the cluster-heads election phase consists of two local diffusion. In the first diffusion, each node locally broadcasts its bid (i.e., nodes competition to become clusterhead). In the second diffusion, nodes elected cluster-heads share their status using corresponding competition radius range.

\section{Enhanced Unequal Clustering Algorithm}

In this paper, we propose Enhanced Unequal Clustering Algorithm (EUCA) that eliminates the first local diffusion performed on UCA (i.e., nodes competition).

At the beginning, the sink launches the first round by broadcasting a message NEW_ROUND_MSG which contains the parameters $d_{\min }$ and $d_{\max }$. Every node $i$ receives such message set back-off timer denoted by $T(i)$ :

$$
T(i)=\lambda \times T_{\max \text { Delay }}
$$

where $T_{\text {max Delay }}$ is the maximum tolerable delay of the backoff timer; $\lambda$ is random number belonging to the interval $[0,1]$.

At the first round all nodes have the same energy level, therefore the back-off timer is set with random number between 0 and $T_{\text {maxDelay. }}$ A sensor node that does not receive cluster-head notice $\mathrm{CH}$ _NOTIFY_MSG before the expiration of its timer, proclaims himself cluster-head for the first round and informs its neighbors using the competition radius range seen in the formula (3). Otherwise, the node cancels its timer and wait for eventual other notice(s). This technical, allows to avoid $N$ (i.e., number of sensor nodes deployed) exchanging control messages for each clustering process. Each simple node chooses a cluster-head with the largest received signal strength and informs them by sending a JOIN_CLUSTER_MSG. The organization of intra-cluster data transmission is similar to $\mathrm{LEACH}$, each sensor node member transmits the sensed data to its cluster-head according to TDMA scheduling.

For the rounds coming after, EUCA performs the same process except that the back-off timer $T(i)$ for a node $i$ is set in way to be inversely proportional to energy residual:

$$
T(i)=T_{\max \text { Delay }} \times\left(1-\frac{E_{i}}{E_{0}}\right)
$$

where $E_{i}$ is the energy residual of a node $i$ and $E_{0}$ is the initial energy of deployed nodes. Our approach avoid to elect more than one cluster-head in a given neighborhood, thus allows a better distribution of the cluster-heads through the network. This proposition can be applied for any clustering algorithm including a competition step. The algorithm 1 describes this election.

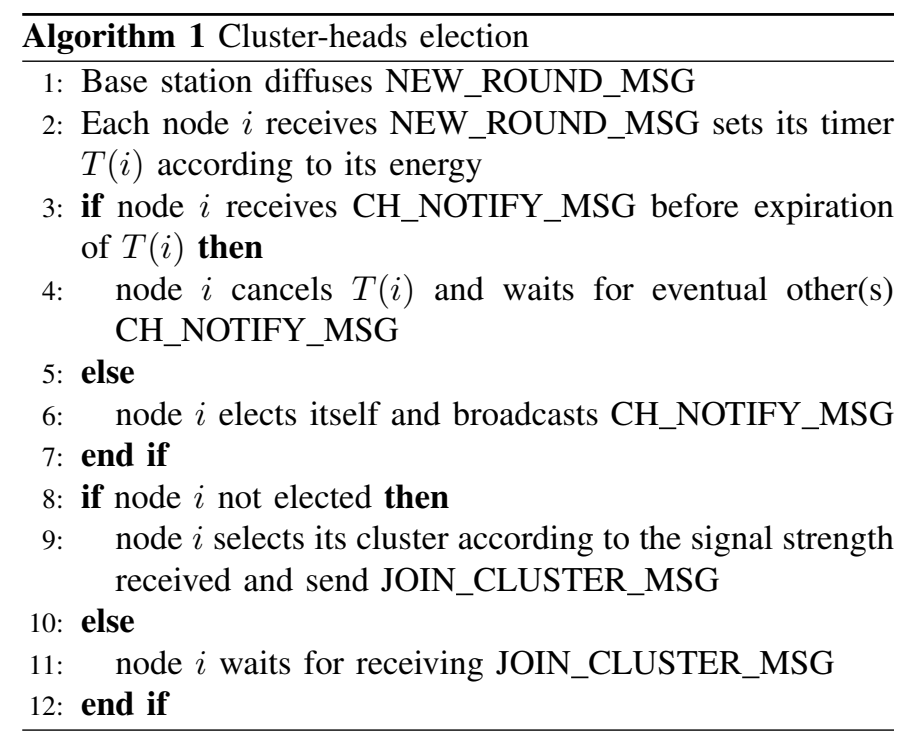

\section{ANALYSIS AND EXPERIMENTAL RESUlT}

\section{A. Communication overhead analysis}

To evaluate the communication overhead when performing the clustering process, we assume that the network is composed of $n$ nodes, and at each round there are an average of $m$ nodes elected as cluster-heads.

In UCA, during the first step, all nodes broadcast their outbid to become cluster-heads which generates $n$ messages. In the second step, nodes elected as headers inform their neighborhood of their new status, thus creating $m$ messages. At the third and final step, nodes that are not elected decide to belong to one of clusters which requires $n-m$ messages. Therefore, the maximum communication overhead introduced by UCA is: $n+m+(n-m)=2 n$.

In EUCA, there is no competition step, each node itself decides its election. Thereby, the maximum of control packets is: $m+(n-m)=n$.

Fig. 3 shows the disparity of communication overhead between EUCA and UCA under network containing 200 sensor nodes, we can clearly observe that the gap between the two costs widens with increasing the number of clustering process (i.e., round).

\section{B. Simulation}

To evaluate the performance of the proposed algorithm EUCA, the simulations are carried out in the network simulator OMNET++ [15], and the UCA is implemented to perform the comparison.The simulation parameters are given in TABLE VI-B, for each experiment, 100 sensors are randomly dispersed into a square of $200 \mathrm{~m} 200 \mathrm{~m}$. The values assigned for the electronic circuits are the same as those MICA2 sensor [16]. The evaluation metric is the amount of energy consumed per node. The result presented in this section is obtained by computing the average of 10 independent experiments for different round duration.

Fig.4 makes the comparison of the average energy spent with different round duration between EUCA clustering approach and UCA. We can see that the amount of energy spent 


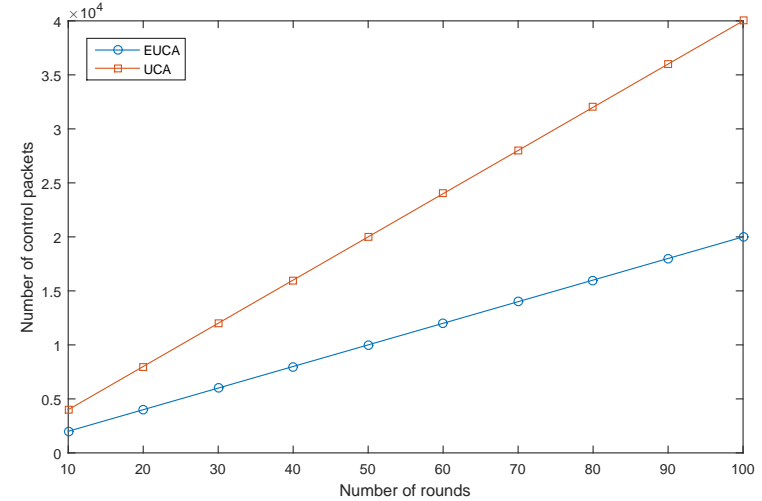

Fig. 3. Communication overhead comparison between EUCA and UCA

TABLE I

SIMULATION PARAMETERS

\begin{tabular}{cc}
\hline Parameters & Values \\
\hline Simulation time & 6000 \\
Network coverage & $200 \mathrm{~m} \times 200 \mathrm{~m}$ \\
Number of nodes & 100 \\
Sink location & $(0,0)$ \\
Control packet size & $10 \times 8$ bits \\
$E_{0}$ & $1000 \mathrm{mj}$ \\
$E_{t r}$ & $50 \mathrm{nj} / \mathrm{bit}$ \\
$T_{\text {max } \text { Delay }}$ & $0.5 \mathrm{~s}$ \\
$\alpha$ & 2 \\
$R_{\max }$ & $50 \mathrm{~m}$ \\
$E_{\text {amp }}$ & $100 \mathrm{pJ} / \mathrm{bit} / \mathrm{m}^{2}$ \\
$d_{\min }$ & $20 \mathrm{~m}$ \\
$d_{\max }$ & $282 \mathrm{~m}$ \\
$k$ & $80 \mathrm{bits}$ \\
$c$ & 0.30 \\
\hline
\end{tabular}

is inversely proportional to the round duration. This is due to the number of performed re-clustering process, more this number is great more is the consumed energy. The graphic shows also that the average energy spent per node is lower in case of EUCA, because the traffic control is reduced on latter by using back-off timer inversely proportional to the node's energy level. This, eliminates the nodes competition during cluster-heads election.

\section{CONCLUSION}

In this paper, an efficient unequal clustering algorithm is introduced to save more energy and bandwidth. This, is achieved by reducing the number of exchanging control messages that time cluster-heads election. The performance evaluation results show that EUCA conserves more energy compared to UCA, thus confirms the contribution of the proposed solution.

As a future work, we will consider the case where multiple adjacent nodes have the same energy level during the cluster- head election step. This case generates collisions that time transmitting the cluster-head notifications (i.e., CH_NOTIFY_MSG) and enable nodes with lower energy to be elected.

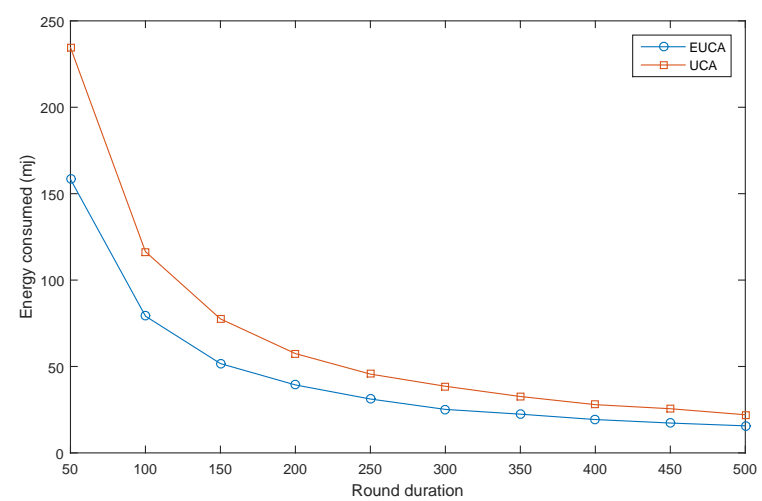

Fig. 4. Average of energy spent with different round duration

\section{REFERENCES}

[1] I. Akyildiz, W. Su, Y. Sankarasubramaniam, and E. Cayirci, "Wireless sensor networks : a survey," Journal of Computer Networks, vol. 38, no. 4 , pp. $393-422,2002$.

[2] G. Anastasi, M. Conti, M. D. Francesco, and A. Passarella, "Energy conservation in wireless sensor networks: A survey," Journal of Ad Hoc Network, vol. 7, pp. 537 - 568, 2009.

[3] G. Gupta and M. Younis, "Load-balanced clustering of wireless sensor networks," in IEEE International Conference on Communication (ICC), vol. 3, 2003, pp. $1848-1852$.

[4] P. Kuila and P. K. Lana, "Improved load balanced clustering algorithm for wireless sensor networks," ADCONS LNCS, vol. 7135, pp. $399-$ 404, 2012.

[5] G. Chen, C. Li, M. Ye, and J. Wu, "An unequal cluster-based routing protocol in wireless sensor networks," Wireless Networks, vol. 15, no. 2, pp. $193-207,2009$

[6] W. Heinzelman, A. Chandrakasan, and H. Balakrishnan, "Energyefficient communication protocol for wireless microsensor networks," in System sciences, 2000. Proceedings of the 33rd annual Hawaii International Conference on. IEEE, 2000, pp. 10-pp.

[7] F. Xiangning and S. Yulin, "Improvement on leach protocol of wireless sensor network," in IEEE, International Conference on Sensor Technologies and Applications, 2007, pp. $260-264$.

[8] M. Sharma and K. Sharma, "An energy efficient extended leach(eee leach)," in IEEE International Conference on Communication Systems and Network Technologies (CSNT), 2012, pp. 377 - 382.

[9] A. Yektaparast, F. H. Nabavi, and A. Sarmast, "An improvement on leach protocol (cell-leach)," in IEEE, 14th International Conference on Advanced Communication Technology (ICACT), 2012, pp. 992 - 996.

[10] O. Younes and S. Fahmy, "Heed : A hybrid, energy-efficient, distributed clustering approach for ad hoc sensor networks," IEEE Transactions on Mobile Computing, vol. 3, no. 4, pp. 366 - 379, 2004.

[11] R. Zhang, Z. Jia, and L. Wang, "A maximum-votes and load-balance clustering algorithm for wireless sensor networks," in IEEE 4th International Conference on Wireless Communications, Networking and Mobile Computing, 2008, pp. $1-4$.

[12] S. B. Ali, K. Ali, and M. A. Lodhi, "Joint event detection \& identification: A clustering based approach for wireless sensor networks," in IEEE Wireless Communications and Networking Conference (WCNC), 2013, pp. $2333-2338$.

[13] T. N. Quynh, T. T. Vinh, and M. B. Quynh, "Multipath routing for cluster-based and event-based protocols in wireless sensor networks," in ACM Proceedings of the Third Symposium on Information and Communication Technology, 2012, pp. 172 - 179.

[14] T. Vinh and M. Takumi, "Adaptive routing protocol with energy efficiency and event clustering for wireless sensor networks," IEICE transactions on communications, vol. 91, no. 9, pp. 2795 - 2805, 2008.

[15] Http://www.omnetpp.org/.

[16] Http://www.xbow.com/. 